\title{
An All-Optical Switching Based on Resonance Breaking With a Transient Grating
}

\author{
Osman Akin and Mehmet Salih Dinleyici
}

\begin{abstract}
A new resonance breaking in-fiber switch as an all-optical network component is investigated and presented. A transient grating is applied to break the transverse resonance of the fundamental waveguide mode and the power coupled into the higher order propagating mode is computed using coupled mode theory (CMT). The coupling of the modes with the grating forming beams in the evanescent region of the waveguide is investigated with four wave mixing (FWM). High conversion efficiency is calculated in the case of perfect phase matching at communication wavelength 1550 $\mathrm{nm}$. The conversion efficiency of the proposed structure is considered in terms of third-order nonlinear susceptibility, and the effect of design and tuning parameters are investigated for grating forming geometry and laser beam intensity, respectively.
\end{abstract}

Index Terms-Four wave mixing (FWM), nonlinear optics, optical Kerr effect, optical switches, transient gratings.

\section{INTRODUCTION}

D RAMATIC increase of data traffic in current communication networks has steered the research interests in photonics into all optical signal processing elements. Accordingly, the invention of high-power lasers made it possible to realize photonic components based on optical nonlinearities requiring high power densities. Designing a faster all-optical switching component using optical nonlinearity is one of the most desired goals in the field of all-optical information processing, and various types of all optical elements have been proposed so far [1], [2]. All optical switches based on semiconductor optical amplifier and Mach-Zender interferometer [3], ring resonator [4] and plasmonics [5] have been demonstrated. However, most of these are far from being satisfactory for the current requirements of optical packet switching, such as switching power requirement, scalability, switching efficiency, and switching rate, which make these components inadequate for future communication networks. The implementation of all optical process of controlling light with light requires nonlinear medium. Although the material nonlinearity observed up to now is still quite low, third-order optical nonlinearity, namely, Kerr effect, is one of the fastest phenomenon that can be exploited to design a high-speed all-optical switching mechanism [6]. Furthermore, the modification of refractive index of Kerr-type medium in free

Manuscript received February 16, 2010; revised May 15, 2010, September 13, 2010; accepted October 10, 2010. Date of publication October 25, 2010; date of current version November 29, 2010. This work was supported by Tubitak under Project 109E240 and IYTE Research Fund under Project 2008IYTE10.

The authors are with Izmir Institute of Technology, Izmir 35430, Turkey (e-mail: osmanakin@iyte.edu.tr; salihdinleyici@iyte.edu.tr).

Color versions of one or more of the figures in this paper are available online at http://ieeexplore.ieee.org.

Digital Object Identifier 10.1109/JLT.2010.2089599 space using holographic method was demonstrated experimentally, and a response time of few femtoseconds was reported [7], [8].

All optical devices comprising various forms of stationary gratings written in core region are widely used in fiber communication and sensing applications. There are numerous publications for all-optical switching based on stationary gratings externally written on the fiber core [9]-[11]. However, Chen et al. discovered that a periodic perturbation created in cladding can affect the fundamental core mode and offer some advantages compared to the traditional methods [12].

In light of these developments, we propose a new in-fiber all optical switching device, which have not been realized yet to our knowledge. This device consists of a transient grating formed by control laser light according to the Kerr effect and another stationary grating placed just after the transient grating. The suggested device is created by partial removal of the cladding and placing a material with high third-order nonlinearity instead of a cladding material. This process was previously investigated experimentally by Dinleyici [13]. The nonlinear medium enables the interaction of propagating mode with the grating forming laser control light that results in all optical switching. The proposed waveguide structure can be analyzed by considering four wave mixing (FWM) of Gaussian beams and propagating mode in the region of core-cladding boundary, where fields of modes and grating forming fields are interacting in the evanescent region of the waveguide and where nonlinear material is placed. Hereafter, the coupled wave equations for the propagation of higher order modes are obtained in the existence of the transient grating. In order to extract these modes out to another waveguide, a second permanent grating may be placed after the transient grating. By using this method, optical data packets can be transferred to another waveguide by means of transient grating and secondary stationary grating. Due to fast response time (fs) of Kerr-type materials, this method gives possibility to achieve high-optical switching rates of about tens of $\mathrm{Gb} / \mathrm{s}$ for recent optical communication systems.

In this method, the existence of transient grating satisfies the switching operation, while the laser beam intensity is the control parameter. The switching efficiency can be controlled via total intensity and the higher Kerr coefficient of the cladding material, the lower intensity is needed to obtain switching operation. Recent developments in polymer science can make it possible to realize this instantaneous switching with low-level control light [14], [15].

In the switching schematic, as shown in Fig. 1, two Gaussian laser beams are interfered at the cladding region of the waveguide to create a transient grating using the Kerr effect. The formed structure is close enough to the core-cladding boundary 


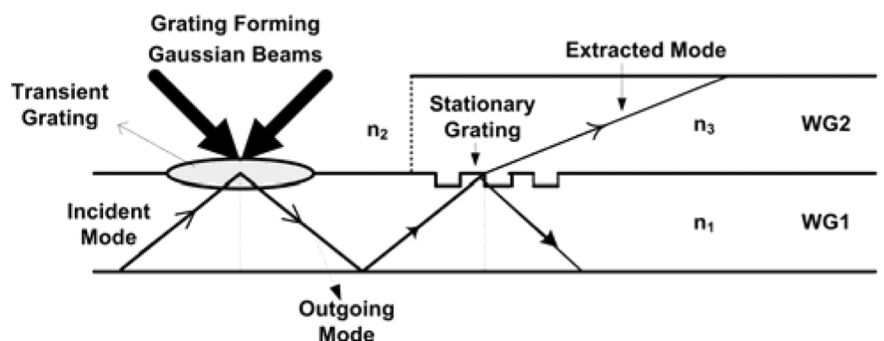

Fig. 1. Schematic of optical switch based on transient grating.

so that it may destroy the guiding properties of the waveguide. In other words, the temporary grating breaks the transverse resonance of the mode and couples the fundamental fiber mode into higher order modes. Thus, this structure can be treated as a photonic crystal with a stopband, allowing the propagation of only certain modes.

In this paper, we investigate the mode switching capability of a transient laser-induced grating formed by interference of Gaussian beams at the core cladding boundary of a waveguide, as shown in Fig. 1. In this study, we are mainly interested in power coupling from fundamental mode into higher order mode due to the FWM interaction and look for the transient grating parameters that maximize the conversion efficiency. Furthermore, we investigate the spectral characteristics of the switch and determine the tuning parameters of the transient grating that affect the transmission efficiency of the whole system.

\section{FWM INTERACTION OF OpticAl GUIDED Modes WiTH CONTROL BEAMS}

\section{A. FWM and Grating Formation}

Consider a two-mode slab waveguide with a cladding composed of third-order nonlinear material. The fundamental mode propagating in the waveguide is represented by $U_{3}$ with a propagation constant $\beta_{3}$ and higher order mode $U_{4}$ with propagation constant $\beta_{4}$. Furthermore, the grating forming Gaussian beams $U_{1}$ and $U_{2}$ at wavelength $\lambda_{L}$ cross at half angle $\theta$ at the cladding side of the core-cladding boundary. The interaction of the propagating mode with the transient grating can be considered as a FWM process in which grating forming beams $U_{1}, U_{2}$ and the propagating waveguide mode $U_{3}$ interact with each other and a fourth wave $U_{4}$ is formed as a result of this FWM interaction.

This interaction permits a real-time ultrafast switching capability considering the outward mode properties. Fig. 2 shows the geometry of the interaction and the fields that are involved in the FWM process. For the waves to be coupled, frequency and phase matching requirements are given by

$$
\begin{aligned}
\omega_{1}+\omega_{3} & =\omega_{2}+\omega_{4} \\
\vec{k}_{1}+\vec{k}_{3} & =\vec{k}_{2}+\vec{k}_{4}
\end{aligned}
$$

where $\omega_{1}=\omega_{2}$ and $\omega_{3}=\omega_{4}$.

Because the light-matter interaction in this case results in a small index perturbation, the overall interaction process in the cladding can be analyzed using coupled mode theory (CMT). The detailed derivation of CMT in nonlinear medium is similar to Saleh and Teich[16], Boyd [17], and Yariv [18]. By using

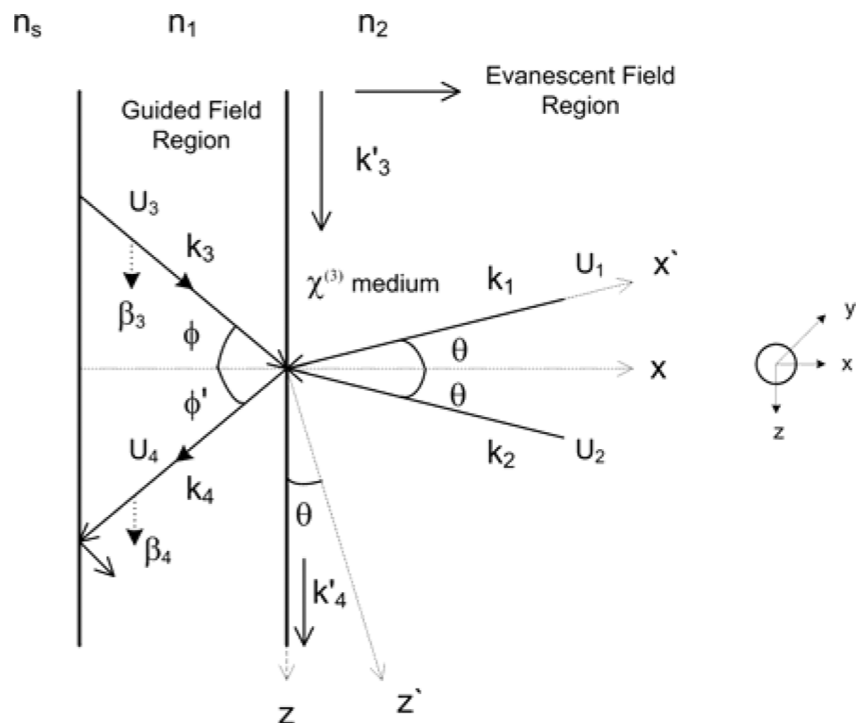

Fig. 2. FWM at the core cladding boundary.

FWM matching conditions given by (1) and (2), the Helmholtz equations for incident and outgoing waves can be written as follows:

$$
\begin{aligned}
& \left(\nabla^{2}+k_{3}^{2}\right) U_{3}=-\zeta_{3} \Psi U_{4}-\gamma_{3} U_{3} \\
& \left(\nabla^{2}+k_{4}^{2}\right) U_{4}=-\zeta_{4} \Psi^{*} U_{3}-\gamma_{4} U_{4}
\end{aligned}
$$

where $\zeta_{3,4}=6 \mu_{0} \omega_{3,4}^{2} \chi^{(3)}, \gamma_{3,4}=2 n_{c l} n_{2}\left(\omega_{3,4}^{2}\right) /\left(c_{o}^{2}\right)(2 I-$ $\left.I_{3,4}\right)$, in which $n_{c l}$ is the cladding refractive index, $n_{2}$ is the Kerr coefficient, $I=I_{1}+I_{2}+I_{3}+I_{4}$ is the total intensity and $I_{3,4}=\left(\left|U_{3,4}\right|^{2}\right) /\left(2 \eta_{0}\right), \chi^{(3)}$ is the third-order nonlinear susceptibility, and $\Psi=U_{1}^{*} U_{2}$, where $U_{1}$ and $U_{2}$ are the grating forming fields of the Gaussian beams.

In the interaction geometry, as shown in Fig. 2, the grating is formed by two Gaussian beams traveling in the $-x^{\prime}$ direction and beams' initial plane is $x^{\prime}=L$. A Gaussian beam can also be expressed in terms of the propagation angle $\theta$ in $(x, y, z)$ coordinate system. Using coordinate transformation and considering the paraxial approximation that involves small intersection angle $\theta$, i.e., $\cos \theta=1$ and $\sin \theta=\theta$, any traveling Gaussian beam can be written as follows:

$$
\begin{aligned}
U=\frac{A}{\rho(x, z, \theta)} \exp (j k \eta) & \\
& \times \exp \left(-j k \frac{\Phi^{2}}{2 \rho(x, z, \theta)}\right) \exp \{-j \omega t\}
\end{aligned}
$$

where

$$
\begin{aligned}
\Phi^{2} & =\mid \begin{array}{ll}
\Phi_{+}^{2}=y^{2}+(x \theta+z)^{2}, & \theta>0 \\
\Phi_{-}^{2}=y^{2}+(-x \theta+z)^{2}, & \theta<0
\end{array} \\
\eta & =\mid \begin{array}{ll}
\eta_{+}=x-z \theta-L, & \theta>0 \\
\eta_{-}=x+z \theta-L, & \theta<0
\end{array} .
\end{aligned}
$$

If $x-L \gg z \theta$, then

$$
\rho=\rho_{+}=\rho_{-}=x-L+j x_{0} .
$$

In these equations, $\mathrm{A}$ is a constant, $x_{0}$ is the Rayleigh range, which is estimated by $\left(\pi / \lambda_{L}\right) W_{0}^{2}, \lambda_{L}$ is the laser wavelength, 
$W_{0}$ is the minimum waist of Gaussian beam, and $\Phi$ is the radial distance.

Assuming that both grating writing beams are originating from the same source; their wavelengths, beam magnitudes, and waists can be considered equal. Although the interference equation consists of traveling and standing waves, a grating pattern is created by the stationary wave pattern given as [19], [20]

$$
\begin{aligned}
\Psi(x, y, z)=\frac{A^{2}}{|\rho|^{2}} & \exp \left(-k_{L} x_{0} \frac{y^{2}+(x \sin (\theta))^{2}+z^{2}}{|\rho|^{2}}\right) \\
& \times \cos \left[2 k_{L} \sin \theta\left(1-x \frac{x-L}{|\rho|^{2}}\right) z\right]
\end{aligned}
$$

where $|\rho|^{2}=\left|x^{\prime \prime}-L\right|^{2}+\left|x_{0}\right|^{2}, k_{L}=(2 \pi) /\left(\lambda_{L}\right)$, and $x^{\prime \prime}$ is the intersection plane $x=x^{\prime \prime}$. The exponential part of this equation defines a Gaussian profile for the grating. The coefficient $k_{L}\left(x_{0}\right) /\left(|q|^{2}\right)$ acts like a diminishing factor in three dimensions, while grating period in the $z$-direction depends on the intersection angle $\theta$, writing beam wavelength $\lambda_{L}$, and the interference plane $(x)$. When the beam interference occurs at the plane $x=0$, the grating period along $z$-direction can be written as $\Lambda=\left(\lambda_{L}\right) /(2 \sin (\theta))$; however, the length of the grating pattern is bounded by the envelope function $-k_{L} x_{0}\left(z^{2}\right) /\left(|\rho|^{2}\right)$, which is very convenient for tuning purposes.

\section{B. Mode Conversion Switching by Means of Transient Grating}

The total field in the interaction region can be written as the superposition of the ideal modes, as follows

$$
\begin{aligned}
U_{3} & =\sum_{m} A_{m}(z) E_{m}(x, y) \exp \left\{-j \beta_{m} z\right\} \\
U_{4} & =\sum_{n} B_{n}(z) E_{n}(x, y) \exp \left\{-j \beta_{n} z\right\}
\end{aligned}
$$

where $A_{m}$ and $B_{n}$ are slowly varying amplitudes. $E_{m}$ is the transverse field distribution of incident (fundamental) mode $(\mathrm{m})$ and $E_{n}$ is the transverse field distribution of the output(higher order) mode (n).

When we substitute (7) and (8) into (3) and (4), we obtain coupled mode equations for every mode:

$$
\begin{aligned}
\frac{d A_{m}}{d z}= & -j \mathrm{~K}_{m}(z) B_{n}(z) \exp \left\{-j\left(\beta_{n}-\beta_{m}\right) z\right\} \\
& +j\left(-\frac{\gamma_{3}}{2 \beta_{m}}\right) A_{m}(z) \\
\frac{d B_{n}}{d z}= & -j \mathrm{~K}_{n}(z) A_{m}(z) \exp \left\{j\left(\beta_{n}-\beta_{m}\right) z\right\} \\
& +j\left(-\frac{\gamma_{4}}{2 \beta_{n}}\right) B_{n}(z)
\end{aligned}
$$

where $K_{m}$ and $K_{n}$ are the mode coupling coefficients denoted as

$$
\begin{aligned}
K_{m}(z) & =\frac{\left|\beta_{m}\right|}{\beta_{m}} \frac{1}{4 \omega \mu} \zeta_{3} C_{m n}(z) \\
K_{n}(z) & =\frac{\left|\beta_{n}\right|}{\beta_{n}} \frac{1}{4 \omega \mu} \zeta_{4} C_{n m}(z)
\end{aligned}
$$

and $C_{n m}$ is the overlap integral coefficient, which can be written as

$$
C_{n m}(z)=\iint_{S} \Psi(x, y, z) E_{m}(x, y) E_{n}^{*}(x, y) d x d y
$$

where $\Psi(x, y, z)$ is the corrugation (grating) function that disrupts the orthogonality of transverse fields. This function results from the interference of Gaussian beams described in (6).

The exact solution of coupled wave (9) and (10) is possible only for uniform grating structures. Nonuniform gratings can be analyzed using numerical approximations. Generalized transfer matrix method [21], effective index method [22], Bloch wave analysis [23], Rouard's method [24] are the best known approaches for analyzing nonuniform grating structures. In this study, we prefer expanding the nonuniform grating function into Fourier series. Considering the length of the grating as $L_{g}$, grating depth as $L_{\text {int }}$ and the period of the grating in the $z$-direction as $\Lambda=\left(\lambda_{L}\right) /(2 \sin \theta)$, the waveguide corrugation function can be written as

$$
\Psi(x, y, z)=\frac{A^{2}}{|\rho|^{2}} \exp \left(-k_{L} x_{0} \frac{y^{2}}{|\rho|^{2}}\right) \mathrm{H}(x, z)
$$

where $H(x, z)$ can be expressed in terms of Fourier series as

$$
H(x, z)=\sum_{p, r} F_{p, r} \exp \left(i \frac{2 \pi p}{L_{g}} z\right) \exp \left(i \frac{2 \pi r}{L_{\mathrm{int}}} x\right) .
$$

Fourier coefficient $F_{p, r}$ is the solution to the integral

$$
\begin{aligned}
& F_{p, r} \\
& =\frac{1}{4 L_{g} L_{\text {int }}} \int_{-L_{g}}^{L_{g}} \int_{-L_{\text {int }}}^{L_{\text {int }}} \exp \left(-k_{L} x_{0} \frac{(x \sin (\theta))^{2}+z^{2}}{|\rho|^{2}}\right) \\
& \quad \times \cos \left[2 k_{L} \sin \theta\left(1-x \frac{x-L}{|\rho|^{2}}\right) z\right] \exp \left(-i \frac{2 \pi r}{L_{\text {int }}}\right) \\
& \quad \times \exp \left(-i \frac{2 \pi p}{L_{g}} z\right) d x d z .
\end{aligned}
$$

The definition of the interaction length $L_{\text {int }}$ of the beams can be found in [25], where it is given by $L_{\text {int }}=\left(W_{0}\right) /(\theta)=$ $\left(\pi W_{0}^{2}\right) /\left(N_{f} \lambda_{L}\right)$ and $N_{f}$ is the number of interference fringes.

Considering the solution of the coupled (9) and (10) for each harmonic $q$ corresponding to each Fourier coefficient parameter $(p, r)$ related to the grating function (12), total amplitudes of the propagating modes in the waveguide can be written as follows:

$$
\begin{aligned}
& A_{m}(z)=\sum_{q} A_{q}(z)=\sum_{p, r} A_{p, r}(z) \\
& B_{n}(z)=\sum_{q} B_{q}(z)=\sum_{p, r} B_{p, r}(z) .
\end{aligned}
$$

Applying boundary conditions $A(0)=A_{0, p, r}, B(0)=0$ and assuming that total optical power is conserved within the 
waveguide, i.e., $\left[(d) /(d z)\left(\left|A_{m}\right|^{2}+\left|B_{n}\right|^{2}\right)=0\right]$, the solution for each harmonic can be written as follows:

$$
\begin{aligned}
A_{p, r}(z)= & {\left[A_{0, p, r} \cos \left(Q_{p, r} z\right)+j \frac{A_{0, p, r}}{Q_{p, r}} \widehat{\delta}_{p, r} \sin \left(Q_{p, r} z\right)\right] } \\
& \exp \left(-j \delta_{p, r} z+j \frac{\alpha_{a}+\alpha_{b}}{2} z\right) \\
B_{p, r}(z)= & -j \frac{K_{n, p, r}}{Q_{p, r}} A_{0, p, r} \sin \left(Q_{p, r} z\right) \\
& \times \exp \left(j \delta_{p, r} z+j \frac{\alpha_{a}+\alpha_{b}}{2} z\right)
\end{aligned}
$$

where $K_{m, p, r}$ and $K_{n, p, r}$ are the amplitudes of coupling coefficients $K_{m(n), p, r}(z) \stackrel{=}{=} K_{m(n), p, r} \exp \left(i\left(2 \pi p / L_{g}\right) z\right)$ for each harmonic of modes $(m)$ and $(n)$, respectively. These coefficients can be written as

$$
\begin{aligned}
K_{m, p, r} & =\frac{\left|\beta_{3}\right|}{\beta_{3}} \frac{1}{4 \omega \mu} \zeta_{3} C_{m n, p, r} \\
K_{n, p, r} & =\frac{\left|\beta_{4}\right|}{\beta_{4}} \frac{1}{4 \omega \mu} \zeta_{4} C_{n m, p, r}
\end{aligned}
$$

where $C_{n m, p, r}$ and $C_{m n, p, r}$ are the integral coefficients related with the orthogonality of the propagating modes, and they are given by $\left(C_{n m, p, r}=C_{m n, p, r}\right)$

$$
\begin{aligned}
C_{p, r}=\iint_{S} & \frac{A^{2}}{\rho^{2}} F_{p, r} \exp \left(-k_{L} x_{0} \frac{y^{2}}{|\rho|^{2}}\right) \\
& \times \exp \left(i \frac{2 \pi r}{L_{\mathrm{int}}} x\right) E_{m}(x, y) E_{n}^{*}(x, y) d x d y
\end{aligned}
$$

where $F_{p, r}$ is defined in (14).

Assuming $K_{p, r}=K_{m, p, r}=K_{n, p, r}$, we find the $Q$ parameter as $Q_{p, r}^{2}=K_{p, r}^{2}+\widehat{\delta}_{p, r}^{2}$, where the detuning parameter $\widehat{\delta}_{p, r}$ is written in terms of the phase-matching parameter $\delta_{p, r}$ and self-coupling coefficients $\alpha_{a}$ and $\alpha_{\mathrm{b}}$ as $\widehat{\delta}_{p, r}=\delta_{p, r}+$ $\left(\alpha_{a}-\alpha_{b}\right) /(2)$. Self-coupling coefficients arise from the thirdorder nonlinearity and depend on the Kerr nonlinearity and total intensity $I$. These coefficients can be written as

$$
\alpha_{a, b}=-\frac{2 n_{\mathrm{cl}} n_{2} \frac{\omega_{3,4}^{2}}{c_{0}^{2}}\left(2 I-I_{3,4}\right)}{2 \beta_{3,4}} .
$$

If we assume that grating forming beams are traveling in $x-z$ plane, then the grating profile in $y$-direction has the Gaussian profile centered at $y=0$ and has a solely effect on breaking the mode orthogonality as specified in (20). This is also the case for the $r$-components in $x$-direction, which mainly affect resonance breaking mechanism. Hence, phase-matching parameter $\delta_{p, r}$ can be written in terms of mode propagation constants $\beta_{3}$ and $\beta_{4}$, grating length $L_{g}$, and Fourier coefficient parameter $p$ (pointing spatial frequency along $z$-direction) as follows:

$$
\delta_{p, r}=\frac{1}{2}\left(\beta_{4}-\beta_{3}\right)+\frac{\pi p}{L_{g}}
$$

where $L_{g}$ is the grating length. Considering the mode amplitudes given by (17) and (18), total mode conversion efficiency resulting from transient grating is

$$
\eta=\frac{\left|B_{n}\left(L_{g}\right)\right|^{2}}{\left|A_{m}(0)\right|^{2}}=\sum_{p, r} \frac{\mathrm{K}_{p, r}^{2}}{Q_{p, r}^{2}} \sin ^{2}\left(Q_{p, r} L_{g}\right) .
$$

The maximum efficiency can be obtained when detuning parameter $\widehat{\delta}_{p, r}=0$. Satisfying this condition leads to the following simple relation:

$$
\beta_{4}\left(1+n_{\mathrm{cl}} \frac{\Delta n_{4}}{n_{\text {eff }}^{2}}\right)=\beta_{3}\left(1+n_{\mathrm{cl}} \frac{\Delta n_{3}}{n_{\text {eff3 }}^{2}}\right)-\frac{2 \pi}{L_{g}} p
$$

where $\Delta n_{3,4}=n_{2}\left(2 I-I_{3,4}\right), n_{\text {eff }}$ is the effective refractive index of the related mode, and mode propagation constants can

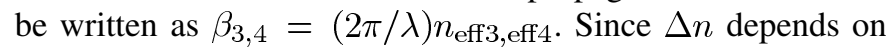
intensity $I$, the conversion process can be tuned by varying the total intensity, and therefore it can be used as a control parameter to provide perfect phase matching. However, interference half angle $\theta$ and material nonlinearity $\chi^{(3)}$ are parameters that can be used as design parameters to obtain high-index variation $\Delta n$. Assuming that phase-matching requirement is met, then maximum efficiency can be obtained by satisfying $Q_{p, r} L_{g}=$ $K_{p, r} L_{g}=(\pi / 2)$ for each component of the mode.

\section{RESULTS}

Consider an asymmetric slab waveguide that allows only two modes to propagate at communication wavelength $\lambda=1550$ $\mathrm{nm}$. Assume that cladding is made of polymeric nonlinear material with refractive index $n_{\mathrm{cl}}=2.32$ and the core refractive index is $n_{\text {core }}=2.4$. Using the dispersion relation for planar dielectric waveguide given in [16], the propagation constants of fundamental mode and higher order mode are calculated as $\beta_{3}=9.708 \times 10^{6}$ and $\beta_{4}=9.6 \times 10^{6}$ for $\omega=1203.2 \times 10^{12}$ $\mathrm{rad} / \mathrm{s}$. By taking the slab thickness as $2 \mu \mathrm{m}$, only two modes are allowed to propagate and introducing a cladding thickness of $750 \mu \mathrm{m}$, approximately $4 \%$ of the total power flows in the cladding region.

The grating profile formed by the interference of Gaussian beams in the cladding of the waveguide is illustrated in Fig. 3. This grating is formed by laser beams of wavelength $532 \mathrm{~nm}$ and an intersection half angle of $\theta=0.6^{\circ}$. The beam minimum waist is $W_{0}=1 \mathrm{~mm}$ and the distance between laser minimum waist location and intersection point is $L=1 \mathrm{~mm}$. The period of the resulting grating is $\Lambda=25.4 \mu \mathrm{m}$. This structure can also be regarded as a 1-D periodic medium placed in the waveguide cladding, where refractive indexes of the successive stacks are $n_{\mathrm{cl}}$ and $n_{\mathrm{cl}}+\Delta n$.

When no grating is present in the proposed structure, most of the power is concentrated in the fundamental mode. The formation of index variation at the cladding region yields a power exchange between the two existing modes that can be analyzed using CMT. In other words, the formation of the grating causes a periodic structure at the cladding that produces a forbidden region for the propagating modes. If the cladding index variation is $\Delta n=0.007$, the dispersion relation provides the upper and 


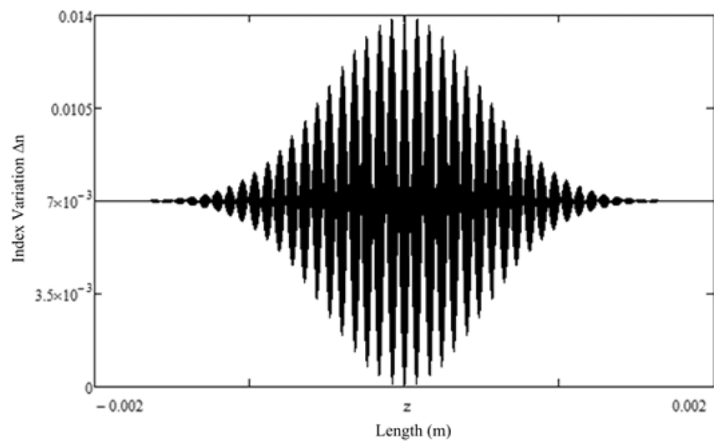

(a)

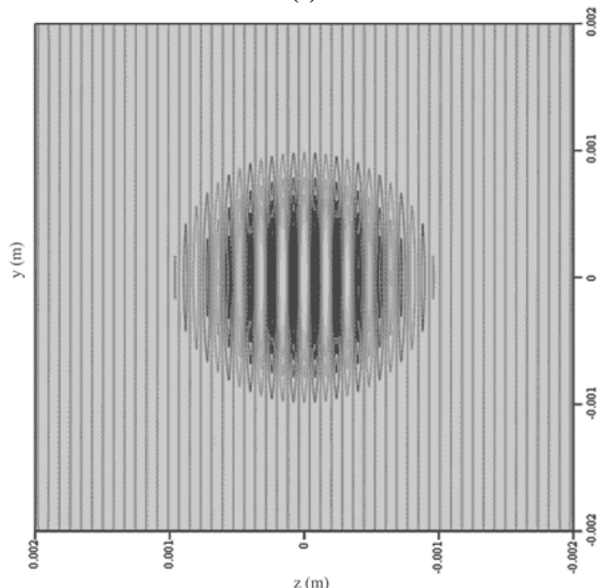

(b)

Fig. 3. (a) 1-D intensity-dependent refractive index pattern. (b) Contour plot of interference fringes.

the lower frequency limits of the forbidden gap, which lies between $11994.05 \times 10^{12} \leq \omega_{B} \leq 1200.167 \times 10^{12}$. In this band structure, the spatial width of the layers is $\Lambda / 2$ and the index difference between the layers is equal to the amplitude of the transient grating, i.e., $\Delta n$. The relation of the mode propagation constants with the band structure is shown in Fig. 4. As can be seen, the propagation constant of the fundamental mode $\beta_{3}$ falls into the local forbidden region, thus in existence of a transient grating it is not allowed to propagate. In contrary, higher order mode propagation constant $\beta_{4}$ is out of the bandgap regime, i.e., it lies on the dispersion line and it is in the propagation regime.

The amplitudes of the fundamental mode $(m)$ and the higher order mode ( $n$ ) are governed by (9) and (10). By expanding the grating function into a 2-D Fourier series along $x$ and $z$ directions, as given by (12), (13), (14), and assuming effective grating length $L_{g}=1 \mathrm{~mm}$, the Fourier coefficients $F_{p, r}$ and corresponding harmonics can be found easily. For the harmonics that contain at least $4 \%$ of the power acquired at the fundamental components of the series, there exist four main components for $z$ direction with spatial frequencies $f_{t}=\left(p_{t}\right) /\left(L_{g}\right)$, where $t=1,2,3,4$ and coefficients $p_{1}=78, p_{2}=79, p_{3}=80$, and $p_{4}=81(0 \leq p, r \leq 100)$. These values are obtained for $N=$ $($ grating length $) /($ grating period $)=(1 \mathrm{~mm}) /(25.4 \mu \mathrm{m}) \cong$ 39 , while for different lengths and periods, the power would be distributed into other harmonics.

When the perfect phase matching condition is satisfied (detuning parameter $\widehat{\delta}_{p, r}=0$ ), a power conversion efficiency of

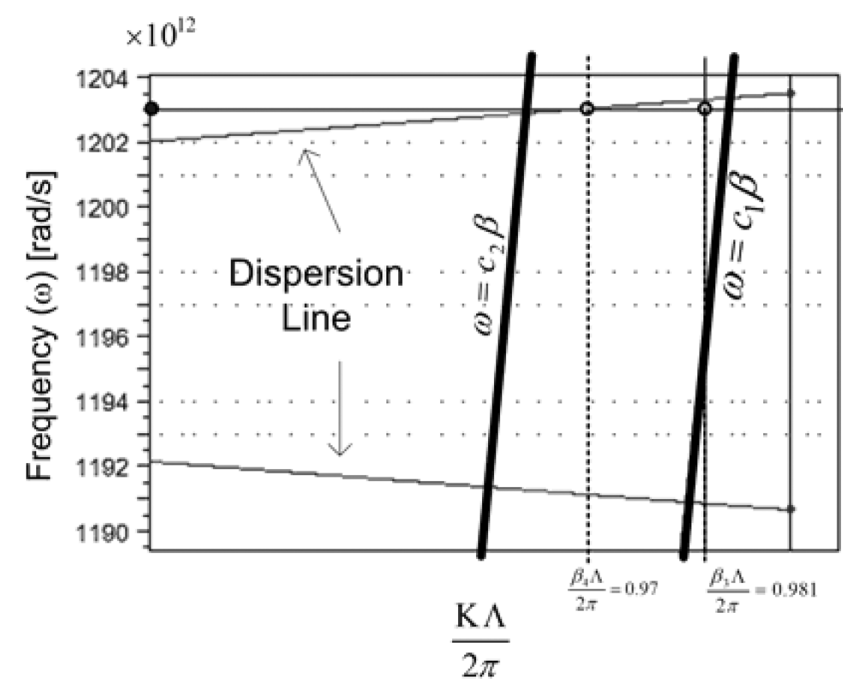

Fig. 4. Dispersion diagram of the periodic medium placed in the evanescent region, where the refractive indexes of successive stacks are $n_{\mathrm{cl}}=2.32$ and $n_{\mathrm{cl}}+\Delta n=2.327$.

about $67 \%$ is obtained at the end of the grating. Such an interaction is not so sensitive to the grating length, which allows for relaxation in critical length adjustment.

The perfect phase matching condition is given by (24), where intensity can be regarded as the phase-matching tuning parameter due to its effect on $\Delta n$. Because of the limited variation in $\Delta n$, it is not an easy task to provide steady perfect phase matching. In this case, if the magnitude of the coupling coefficient is comparable to the magnitude of the phase mismatch (detuning parameter $\widehat{\delta}$ ), the coupling between the propagating modes is limited and most of the power remains in the fundamental mode. In this situation, the coupling efficiency remains at about 33\%, as shown in Fig. 5.

Another way of getting strong coupling into higher order mode is to ensure over coupling, i.e., $|\mathrm{K}| \gg|\widehat{\delta}|$. In such a case, an instantaneous power coupling occurs at the beginning of the transient grating and there exist some fluctuations through the grating leading to an average conversion efficiency of about $48 \%$, calculated at the end of the grating, as shown in Fig. 6.

On the other hand, third-order optical nonlinearity is another important factor that has a major influence on mode conversion efficiency. In Fig. 7, we depict the coupling efficiency with respect to nonlinearity for both transient nonuniform and transient sinusoidal gratings and compare the efficiency for different type of nonlinear materials and phase matching situations. The third-order nonlinear susceptibility of the optical glasses is typically about $(1-100) \times 10^{-14}$ esu [17], while this value is in the order of $(2.4 \pm 0.8) \times 10^{-13}$ esu for Lithium Niobate [26] and about $2 \times 10^{-7}$ esu for polymers [27]. If we take a closer look at the relation between power coupling efficiency and third-order nonlinear susceptibility for the transient grating formed in optical glasses $\left(\chi^{(3)} \leq 10^{-12}\right)$, we see that the coupling efficiency is much higher for both sinusoidal grating and nonperiodic grating if the phase matching condition is satisfied. A further increase in the susceptibility (using polymer with susceptibility of about $10^{-7}$ esu instead of optical glass) enhances the efficiency of the nonperiodic grating. 


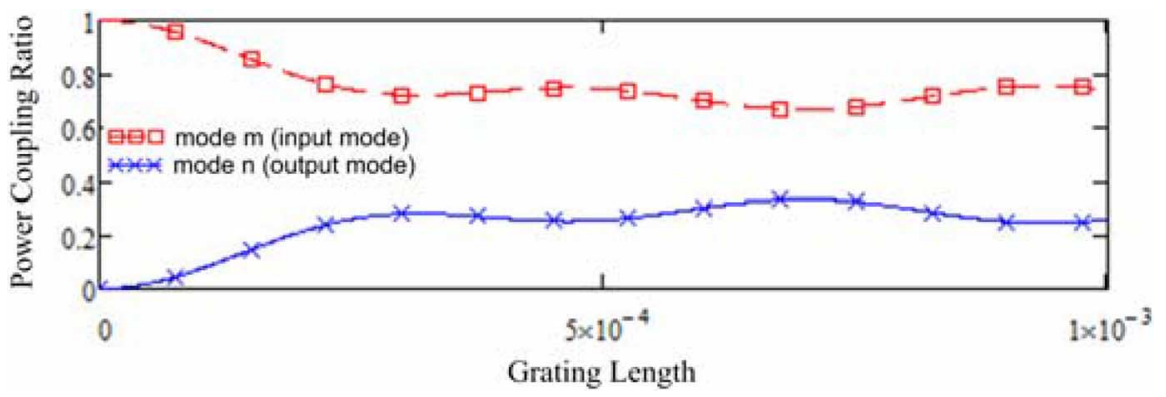

Fig. 5. Input and output power in the case of under coupling (no phase matching: $\mathbf{K} \cong \widehat{\delta}$ ).

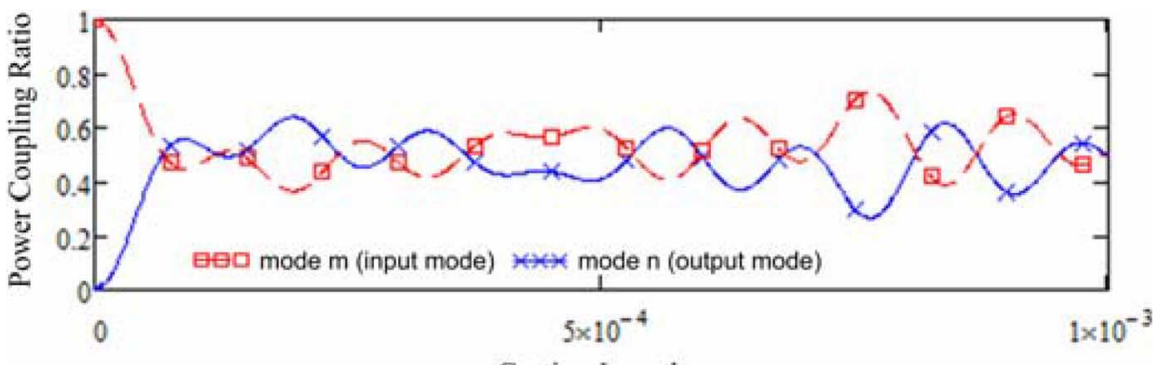

Grating Length

Fig. 6. Power coupling in the case of over coupling $(|\mathbf{K}| \gg|\widehat{\delta}|)$.

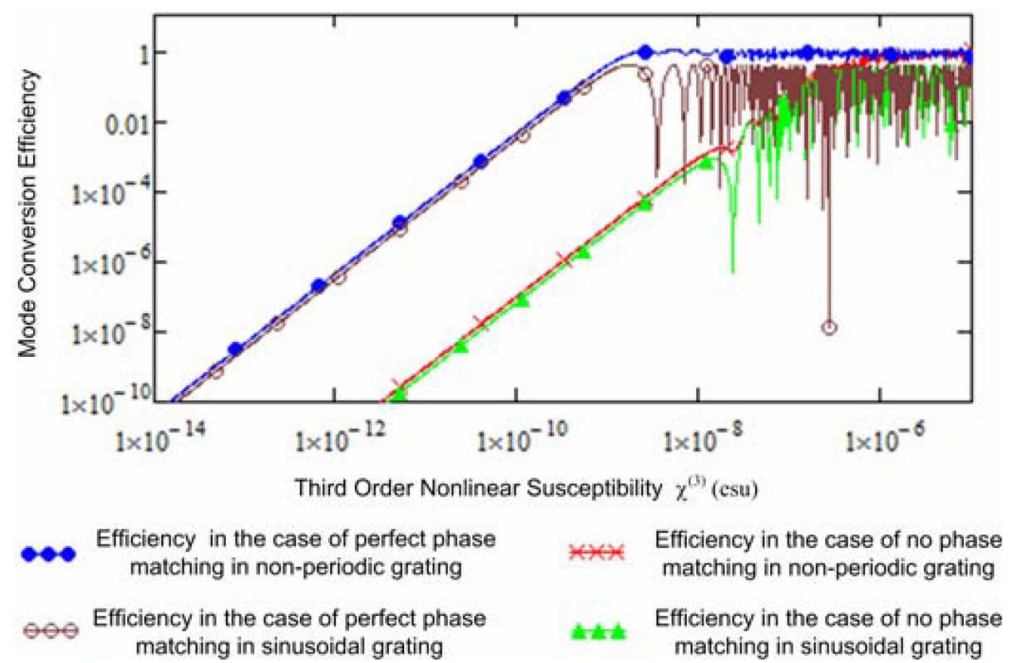

Fig. 7. Graphical representation of efficiency versus third-order optical susceptibility (grating length constant).

When the susceptibility is below some threshold value (about $10^{-9}$ ), there is a linear relation between nonlinearity and efficiency. Efficiency reaches saturation for the nonuniform grating and gets nearly constant values above the threshold, while there are large efficiency fluctuations for sinusoidal gratings. When the nonlinearity is weak, the satisfaction of phase matching is the fundamental factor that has an influence on the efficiency, but a further increase in the nonlinearity may result in over coupling, i.e., $|\mathrm{K}| \gg \widehat{\delta}$, which diminishes the effect of phase matching.

Considering the spectral characteristics of the grating, the maximum power coupling between the modes occurs in the case of perfect phase matching at the wavelengths satisfying (24). Alternatively, maximum coupling occurs when

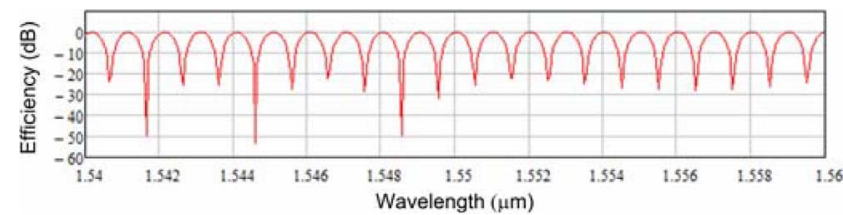

Fig. 8. Spectral characteristic of the grating.

$Q_{p, r} L_{g}=(\pi) /(2)$, which can be optimized for different wavelengths by choosing appropriate design parameters (interference half angle, grating period) and waveguide parameters. Normalized spectral characteristics of the non-uniform grating for phase-mismatched coupling $\left(\widehat{\delta}_{p, r} \neq 0\right)$ with the parameters given above is shown in Fig. 8. 
For the selected set of parameters, there is a 1-nm spacing between the maximum conversion peaks in the spectrum. In contrary, there are deeps at wavelengths 1541.6, 1544.5, and $1548.5 \mathrm{~nm}$, where the power efficiency drops about $40 \mathrm{~dB}$, and the mode coupling is completely suppressed. Using the tuning parameters of the transient grating such as interference angle and laser wavelength, the efficiency can be maximized for the desired modes. This optimization will ensure the utilization of the system in dense wavelength-division-multiplexing (DWDM) applications such as a tunable mode coupler or drop filter.

\section{CONCLUSON}

A new resonance breaking in-fiber network switch is introduced and analyzed with FWM and CMT. This type of a device seems especially promising for all-optical network switches, routers, and DWDM devices, where the optical packets need to be switched or routed in fiber among a few output ports. Since the resonance breaking is one of the fastest mechanisms for optical switching, in this study, it is combined with Kerr-type nonlinearity for all optical function, and also in order to improve switching power requirements while keeping switching speed high.

Switching power is crucial for Kerr-type nonlinear integrated optical switching devices when the optical control signal is not very strong. In this paper, the transverse resonance of the fundamental mode is broken by the transient grating, which corresponds to the stopband of the 1-D photonic bandgap material (PBG). Therefore, the modal power spreads into higher order modes, which are allowed to propagate in PBG. The resonance quality of the fiber cavity and setting the fundamental mode close to cut off is very important in terms of minimum power requirement as well as switching response time.

The switching response time fundamentally depends on the nonlinear material response time, which is reported to be $10^{-15}$ $\mathrm{s}$ for Kerr-type nonlinear glasses due to electronic transitions [6], [8]; therefore, the switching time is virtually limited only by resonance breaking dynamics; which is not included and estimated in the study.

The simulations presented in this paper show that $67 \%$ mode power coupling can be achieved without critical length adjustment under perfect phase matching condition. However, for the lack of phase matching, the power coupling is around 33\%, as shown in Fig. 5. The model used in the simulation also shows that the phase matching can be tuned to match by means of the total power in the grating forming beams.

If the device performance is compared with transient sinusoidal grating, as shown in Fig. 7, the proposed device performs better both at low and high nonlinear susceptibilities. Furthermore, it is not oscillating at all, which confirms inessentiality of the critical length.

Although the device is designed to switch at $1550 \mathrm{~nm}$, the spectral characteristics show that it has 1-nm separated channels. This makes the device very attractive for applications like tunable filters; add/drop channels for DWDM and wavelength recognizing switching, etc. The proposed device, therefore, seems promising indeed for a practical implementation of all-optical network switches/routers or add/drop filter for DWDM networks.

\section{REFERENCES}

[1] T. S. El-Bawab, Optical Switching. New York: Springer-Verlag, 2006.

[2] A. M. C. Dawes, D. J. Gauthier, S. Schumacher, N. H. Kwong, R. Binder, and A. L. Smirl, "Transverse optical patterns for ultra-low-light level all-optical switching," Laser Photon. Rev., vol. 4, pp. 221-221, 2009.

[3] V. Lal, M. L. Masanovic, J. A. Summers, G. Fish, and D. J. Blumenthal, "Monolithic wavelength converters for high-speed packet-switched optical networks," IEEE J. Sel. Topics Quantum Electron., vol. 13, no. 1, pp. 49-49, Jan./Feb. 2007.

[4] A. Martinez et al., "Ultrafast all-optical switching in a silicon-nanocrystal-based silicon slot waveguide at telecom wavelengths," Nano Lett., vol. 10, pp. 1506-1511, 2010.

[5] A. Y. Elezzabi, Z. Han, S. Sederberg, and V. Van, "Ultrafast all-optical modulation in silicon-based nanoplasmonic devices," Opt. Exp., vol. 17, no. 13, pp. 11045-11045, Jun. 2009.

[6] T. Schneider, D. Wolfframm, and J. Reif, "Ultrafast laser-induced index grating in transparent insulators," Nucl. Instrum. Methods Phys. Res. Section B Beam Interactions Mater. Atoms, vol. 166-167, pp. 809-814, May 2000.

[7] H. T. Hsieh, D. Psaltis, O. Beyer, D. Maxein, C. Schmising, K. Buse, and B. Sturman, "Femtosecond holography in lithium niobate crystals," Opt. Lett., vol. 30, pp. 2233-2233, 2005.

[8] T. Schneider, D. Wolfframm, R. Mitzner, and J. Reif, "Ultrafast optical switching by instantaneous laser-induced grating formation and selfdiffraction in barium fluoride," Appl. Phys. B, Lasers Opt., vol. 68, no. 4, pp. 749-751, Apr. 1999.

[9] B. J. Eggleton, R. E. Slusher, J. B. Judkins, J. B. Stark, and A. M. Vengsarkar, "All-optical switching in long-period fiber gratings," Opt. Lett., vol. 22, no. 12, pp. 883-885, Jun. 1997.

[10] K. O. Hill, B. Malo, K. A. Vineberg, F. Bilodeau, D. C. Johnson, and L. Skinner, "Efficient mode conversion in telecommunication fibre using externally written gratings," IEEE Electronics Letters, vol. 26, no. 16, pp. 1270-1270, Aug. 1990.

[11] I. V. Kabakova, B. Corcoran, J. A. Bolger, C. M. de Sterke, and B. J. Eggleton, "All-optical self-switching in optimized phase-shifted fiber Bragg grating," Opt. Exp., vol. 17, no. 7, pp. 5083-5083, Mar. 2009.

[12] N. Chen, B. Yun, and Y. Cui, "Cladding index modulated fiber grating," Opt. Commun., vol. 259, pp. 587-587, 2006.

[13] M. S. Dinleyici, "An experimental work on optical component based on D-fiber/slab evanescent coupling structure," Opt. Quantum Electron., vol. 35, pp. 75-84, 2003.

[14] P. Poornesh, P. K. Hegde, G. Umesh, M. G. Manjunatha, K. B. Manjunatha, and A. V. Adhikari, "Nonlinear optical and optical power limiting studies on a new thiophene-based conjugated polymer in solution and solid PMMA matrix," Opt. Laser Technol., vol. 42, pp. 230-236, 2010.

[15] S. A. Haque and J. Nelson, "Toward organic all-optical switching," Science, vol. 327, no. 5972, pp. 1466-1467, Mar. 2010.

[16] B. E. A. Saleh and M. C. Teich, Fundamentals of Photonics. New York: Wiley, 2007.

[17] R. W. Boyd, Nonlinear Optics. San Diego, CA: Academic, 1992.

[18] A. Yariv, "Coupled-mode theory for guided-wave optics," IEEE J. Quantum Electron., vol. QE-9, no. 9, pp. 919-933, Sep. 1973.

[19] M. S. Dinleyici and O. Akin, "3-D switching fabric node design," presented at the IASTED Communications Systems and Networks, Spain, Sep. 1-3, 2004.

[20] O. Akin, "All-optical switching via diffraction grating formed by interference of Gaussian beams," M.Sc. thesis, Izmir Inst. Technol., Izmir, 2005.

[21] M. Yamada and K. Sakuda, "Analysis of almost-periodic distributed feedback slab waveguides via a fundamental matrix approach," Appl. Opt., vol. 26, pp. 3474-3478, 1987.

[22] K. A. Winick, "Effective-index method and coupled-mode theory for almost-periodic waveguide gratings: A comparison," Appl. Opt., vol. 31, no. 6, pp. 757-764, Feb. 1992.

[23] E. Peral and J. Capmany, "Generalized Bloch wave analysis for fiber and waveguide gratings," J. Lightw. Technol., vol. 15, no. 8, pp. 1295-1302, Aug. 1997.

[24] L. A. Weller-Brophy and D. G. Hall, "Analysis of waveguide gratings: Application of Rouard's method," J. Opt. Soc. Amer. A, vol. 2, no. 6, pp. 863-871, Jun. 1985. 
[25] A. E. Siegman, "Bragg diffraction of a Gaussian beam by a crossed-Gaussian volume grating," J. Opt. Soc. Amer., vol. 67, no. 4, pp. 545-550, Apr. 1977.

[26] A. Kulagin et al., "Components of the third-order nonlinear susceptibility tensors in KDP, DKDP and LiNbO nonlinear optical crystals," Quantum Electron., vol. 34, no. 7, p. 657, Jul. 2004.

[27] X. Zhan, Y. Liu, D. Zhu, X. Liu, G. Xu, and P. Ye, "Large third-order nonlinear optical response of a conjugated copolymer consisting of 2,5diethynylthiophene and carbazole units," Chem. Phys. Lett., vol. 343, no. 5-6, pp. 493-498, Aug. 2001.

Osman Akin received the B.S. degree in electrical and electronics engineering from Ege University, Izmir, Turkey, the M.S. degree of electronics engineering from Izmir Institute of Technology, Izmir, Turkey, where he is currently working toward the Ph.D. degree.

His current research interests include photonic switching systems and nonlinear optics.
Mehmet Salih Dinleyici received the B.S. and M.S. degrees from the Electrical and Electronics Engineering Department, Middle East Technical University, Ankara, Turkey, in 1989 and 1992, respectively, and the Ph.D. degree in electrical engineering from Illinois Institute of Technology, Chicago, IL, in 1998.

Since 2006, he has been an Associate Professor in the Electrical and Electronics Engineering Department, Izmir Institute of Technology, Izmir, Turkey, where he is currently engaged in research on photonic structures for optical communication networks. 